Canad. Math. Bull. Vol. 20 (4), 1977

\title{
THE METRIZABILITY OF SPACES WHOSE DIAGONALS HAVE A COUNTABLE BASE
}

\author{
BY \\ JOHN GINSBURG
}

\begin{abstract}
It is shown that the diagonal of $X$ has a countable neighborhood base in $X \times X$ if and only if $X$ is a metrizable space whose set of non-isolated points is compact.
\end{abstract}

The aragonal of $X$ is the set $\Delta_{X}=\{(x, x): x \in X\}$. A family $U$ of open subsets of $X \times X$ is a base for $\Delta_{X}$ in $X \times X$ if every member of $U$ contains $\Delta_{X}$ and every neighborhood of $\Delta_{X}$ in $X \times X$ contains a member of $U$.

Based on a recent result due to Chaber [3] on countably compact spaces with $G_{\delta}$-diagonals, and on Bing's well-known metrization theorem [1], we will give a short proof of the theorem stated in the abstract.

Our topological notation and terminology are standard. We will assume no separation axioms beyond $T_{2}$ of our spaces. Interesting results on neighborhoods of the diagonal can be found in Simon's paper [4] which has helped to motivate our proof.

Recall that, if $\mathscr{G}$ is an open cover of $X$ and $p \in X$, then the star of $p$ with respect to $\mathscr{G}$, denoted by $s t(p, \mathscr{G})$, is the union of all the members of $\mathscr{G}$ which contain $p$.

1. Lemma. Let $X$ be a Hausdorff space and let $\mathcal{U}$ be a base for the diagonal in $X \times X$. For $U \in \mathcal{U}$, we set $\mathscr{G}_{U}=\{G: G$ is open in $X$ and $G \times G \subseteq U\}$. For $U \in \mathcal{U}$ and $S \subseteq X$, we set $U(S)=\{x \in X:(s, x) \in U$ for some $s \in S\}$.

(a) If $A$ is closed in $X$ then $\{U(A): U \in U\}$ is a base for the neighborhoods of $A$ in $X$.

(b) For all $U \in \mathcal{U}, \mathscr{G}_{U}$ is an open cover of $X$ and $s t\left(x, \mathscr{G}_{U}\right) \subseteq U(x)$ for all $x \in X$. Thus $\left\{\mathscr{G}_{U}: U \in \mathscr{U}\right\}$ is a development for $X$.

(c) If $A$ is closed in $X$, then $\{U \cap(A \times A): U \in U\}=U_{A}$ is a base for the neighborhoods of $\Delta_{A}$ in $A \times A$.

Proof. (a) If $G$ is open and $A \subseteq G$, then $(G \times G) \cup(X-A) \times(X-A)$ is a neighborhood of the diagonal, so there is a member $U$ of $U$ such that $U \subseteq(G \times G) \cup(X-A) \times(X-A)$. For such $U$, clearly $U(A) \subseteq G$.

Received by the editors January 4, 1977 and, in revised form, March 17, 1977.

AMS Subj. Class. 54E35.

Key words and phrases: diagonal, neighborhood base, metrizability, compact. 
(b) This result is essentially established in [2], and can be verified in a straight-forward manner. One first shows that $s t\left(x, \mathscr{G}_{U}\right) \subseteq U(x)$. The sets $U(x)$ form a base at $x$ by (a), and therefore so do the sets $\operatorname{st}\left(x, \mathscr{G}_{U}\right)$. Thus $\left\{\mathscr{G}_{U}: U \in \mathscr{U}\right\}$ is a development for $X$.

(c) Let $W$ be open in $A \times A$ such that $\Delta_{A} \subseteq W$. Find an open set $W_{1}$ in $X \times X$ such that $W_{1} \cap(A \times A)=W$. Then $W_{1} \cup(X-A) \times(X-A)$ is a neighborhood of $\Delta_{X}$ in $X \times X$, so there exists $U \in \mathcal{U}$ with $U \subseteq$ $W_{1} \cup(X-A) \times(X-A)$. Intersecting both sides of this inclusion with $A \times A$ gives $U \cap(A \times A) \subseteq W$, as desired.

2. THEOREM. Let $X$ be a Hausdorff space. The diagonal of $X$ has a countable base in $X \times X$ if, and only if $X$ is a metrizable space whose set of non-isolated points is compact.

Proof. Let $I$ be the set of isolated points of $X$, and let $U$ be a base for $\Delta_{X}$ in $X \times X$ such that $|u| \leq \aleph_{0}$. By 1.(a), $X$ is first countable. The standard diagonalization argument which shows that the set of integers does not have a countable base in the real line can be easily extended to show that in a first countable space $Y$, no closed discrete countable set consisting of non-isolated points of $Y$ has a countable base for its neighborhoods in $Y$. Since, by 1.(a), every closed set in $X$ has a countable base in $X$, we see that $X-I$ can contain no closed discrete infinite set. That is, $X-I$ is countably compact. By 1 (c), every closed subset of $X$ has a $G_{\delta}$-diagonal. That is, if $A$ is closed in $X$, then $\Delta_{A}$ is an intersection of countably many open subsets of $A \times A$. By Chaber's theorem [3], countably compact spaces with $G_{\delta}$-diagonals are compact. Applying these remarks to the closed set $X-I$, we see that $X-I$ is compact. Now, it is easy to see that a space which is the union of a set of isolated points and a compact set is paracompact, and so $X$ is paracompact. But, by 1.(b), $X$ has a countable development. So $X$ is a paracompact Moore space, and hence a collectionwise normal Moore space. By Bing's theorem [1], $X$ is metrizable. We omit the elementary verification of the converse.

\section{REFERENCES}

1. R. H. Bing, Metrization of topological spaces, Canadian J. Math. 3 (1951), 175-186.

2. J. G. Ceder, Some generalizations of metric spaces, Pacific J. Math. 11 (1961), 105-126.

3. J. Chaber, Conditions which imply compactness in countably compact spaces, to appear.

4. P. Simon, A note on cardinal invariants of square, Comment Math. Univ. Carolinae 14 (1973), 205-213.

UNIVERSITY OF MANITOBA, Department of MATHEMATiCs, WINNIPEG, MANITOBA. R3T 2N2 